\title{
Odd-Skipped Related Genes Regulate Differentiation of Embryonic Limb Mesenchyme and Bone Marrow Mesenchymal Stromal Cells
}

\author{
Sigmar Stricker, ${ }^{1,2}$ Susanne Mathia, Julia Haupt, ${ }^{1,3}$ Petra Seemann, ${ }^{1,3}$ Julia Meier, ${ }^{1}$ and Stefan Mundlos ${ }^{1-3}$
}

The regulation of progenitor cell differentiation to a specific tissue type is one of the fundamental questions of biology. Here, we identify Osr1 and Osr2, 2 closely related genes encoding zinc finger transcription factors, as being strongly expressed in irregular connective tissue (ICT) fibroblasts in the chicken embryo, suitable as a developmental marker. We provide evidence that both Osr1 and Osr2 regulate mesenchymal cell-type differentiation. Both Osr1 and Osr2 can promote the formation of ICT, a cell type of so far unknown molecular specification, while suppressing differentiation of other tissues such as cartilage and tendon from uncommitted progenitors. Conversely, knockdown of either Osr gene alone or in combination reverses this effect, thereby leading to decreased differentiation of ICT fibroblasts and increased chondrogenesis in vitro. This indicates that Osr genes play a pivotal role in ICT fibroblast differentiation. Undifferentiated mesenchymal cells reside in the adult body in the form of mesenchymal stem cells in the bone marrow cavity. Using bone marrow stromal cells (BMSCs) isolated from chicken fetal long bones, we show that Osr1 and Osr2 have an intrinsic role in BMSC differentiation similar to their role in early embryonic development, that is, the enforcement of CT fibroblast differentiation and the repression of other cell types as exemplified here by osteoblast differentiation.

\section{Introduction}

$\mathrm{M}$ ESENCHYMAL CELLS CONSTITUTE the majority of the vertebrate body including the connective tissues (CTs), muscles, or the hematopoietic system. Thus, during embryonic development, a multitude of different cell lineages and tissue types emerge from these undifferentiated mesenchymal progenitors [1,2].

The CT is an indispensable component for any multicellular organism. Generally, the term "connective tissue" is applied to tissues of mesodermal origin that provide structural and metabolic support and protection for other tissues and organs [3]. The CT proper comprises loose irregular CT (ICT) such as the dermal CT and the muscle CT (MCT). Dense regular CTs comprise tendons and ligaments, whereas cartilage and bone are often referred to as specialized CT. The bulk of CT proper cells present, for example, in the vertebrate limb are ICT cells. However, no general marker for ICT has so far been described, and the knowledge about the differentiation of CT fibroblasts and their maintenance and separation from other tissue types is scarce.

Progress has been made in deciphering the genetic and molecular mechanisms that govern the emergence of differ- ent cell types in vitro as well as in vivo using the mouse or the chick as model systems. The limb bud is an ideal system to analyze the differentiation of mesenchymal cells. Initially, the limb bud consists of undifferentiated mesenchymal cells derived from the lateral plate mesoderm covered by a layer of ectoderm [4]. The ICT, tendons, cartilage, and bone cells arise from these mesenchymal progenitors; whereas muscles and blood vessels originate from cells that migrate into the limb bud from the somites [5-7]. Although the development of muscle, bone, cartilage, and endothelium has been a matter of intense research, little is known about the origin of ICT.

In the adult body, progenitors of mesodermal origin are maintained as stem cells comprising of, for example, hematopoietic stem cells or muscle satellite cells. A special type of stem cells are the mesenchymal stem cells (MSCs) often referred to as skeletal stem cells or also bone marrow stromal cells (BMSCs) $[8,9]$. These cells have retained multipotency and are capable of differentiating into different tissue types as osteoblasts, chondrocytes, adipocytes, or fibroblasts, and others. The BMSCs are promising candidates for regenerative therapies and have been evaluated in different disease contexts in animal models [10-12].

\footnotetext{
${ }^{1}$ Max Planck Institute for Molecular Genetics, Berlin, Germany.

${ }^{2}$ Institute for Medical and Human Genetics, University Medicine Charité, Berlin, Germany.

${ }^{3}$ Berlin-Brandenburg Centre for Regenerative Therapies BCRT, University Medicine Charité, Berlin, Germany.
} 
Here, we identify Odd skipped-related genes Osr1 and Osr2 as factors controlling chicken fetal limb mesenchymal cell differentiation as well as BMSC differentiation. The Osr1 and Osr2 genes encode zinc finger transcription factors that show high sequence similarity between each other are highly conserved among vertebrates [13-17] and appear to act functionally equivalent in the mouse [18]. The founding member of the family, Odd skipped, was first described as a pair-rule gene in Drosophila melanogaster [19]. Both Odd skipped-related genes exhibit a highly dynamic expression pattern during chick and mouse development [13-15]. The Osr1 gene was shown to be necessary for embryonic heart and urogenital development [20-22], whereas Osr2 was involved in secondary palate development [23] and tooth patterning in the mouse [24]. We here show that Osr1 and Osr2 genes are predominantly expressed in ICT cells during chicken fetal limb development. Both Osr1 and Osr2 can drive the differentiation of ICT cells at the expense of other tissues, and they are required for the differentiation of ICT fibroblasts. In BMSCs, we show that both Osr1 and Osr2 are intrinsic negative regulators of osteoblast differentiation and bone formation.

\section{Materials and Methods}

\section{In situ hybridization, immunohistochemistry, and skeletal preparations}

Section and whole-mount in situ hybridization (ISH) using digoxygenin labeled riboprobes was carried out as described in [25]. Fluorescence labeled 2-color ISH was performed as described in [15]. For myosin staining after ISH, sections were permeabilized with $0.1 \%$ Triton $\mathrm{X}-100$ in 5\% bovine serum albumin (BSA). Antibody A4.1025 (Upstate 05-716) against all myosin heavy chain (MyHC) types [26] was applied at 1:200 in the same solution at $4^{\circ} \mathrm{C}$ over night. After washing with phosphate-buffered saline (PBS), secondary antibody (Molecular Probes A11003) was applied in 5\% BSA at 1:500. After washing with PBS, slides were covered with Fluoromount. Skeletal preparations were performed as in [27].

\section{Retroviral constructs, virus production}

Full-length mouse Osr1 and Osr2 [13,14] was cloned by polymerase chain reaction (PCR) using the Primers F: ATACCATGGGCAGCAAAACCTTGCCAG, R: ATAAGA TCTTTAGCATTTGATCTTGGAGGTTTTG and F: ATACC ATGGGGAGCAAGGCCTTGCCAG， R: ATGGATCCTCA GGCTGTGCCGCCGCAGATC. The PCR product was cloned into pSlax13 by NcoI and BglII/BamHI or BamHI and shuttled to RCASBP(A) via ClaI. For the repressor and activator forms of Osr1 and Osr2, the 3' part of the coding sequence containing the nuclear localization signal and the zinc finger domains was amplified with primers F: ATAC CATGGGAAGGTTACCATCCAAAAC and R: ATAGAA TTCTCAGAAGTCCTGCCGCG for Osr1 and F: ATACCAT GGGCCGCCTGCCCTCCAAG, R: ATAGAATTCGAAGTC CTGCCGCG for Osr2. The PCR products were digested with NcoI and EcoRI and cloned into pSlax-Engrailed and pSlaxVP16, respectively, and shuttled to RCAS(BP)A via ClaI.

For knockdown of Osr genes, we employed the replication competent avian retrovirus (RCAS)-based shRNA system developed by Das et al. [28]. For each cOsr1 or cOsr2 (Entrez gene IDs 100316920 and 771523), 3 different target sequences were chosen using the Ambion ${ }^{\text {TM }}$ siRNA target finder and cloned into RCASARNAi according to [28], and knockdown efficacy was tested in chicken micromass (MM) cultures. The 2 most effective hairpin constructs were subsequently used in the experiments. Effective target sequences: cOsr1\#1: GAACTGAAACCTGCCAAACTT, cOsr1\#2: GCACTTCAC CAAGTCGTACAA, cOsr2\#1: GTACATCCATTCCAAAGA GAA, cOsr2\#2: CCACTTCACCAAGTCCTACAA. We used RCAS-sh-Luciferase or RCAS-sh-Gfp as controls [28].

Concentrated viral supernatant was produced as described in Morgan and Fekete [29]. All viral constructs used had a titer of at least $10^{8}$ infectious units per mL. Virus injection into Hamburger and Hamilton ( $\mathrm{HH}) 10$ [30] right hindlimb field was performed according to Logan and Tabin [31].

\section{Cell culture}

The MM cultures and quantification of Alcian blue staining was performed as previously described [32]. For sectioning, MM cultures were embedded in paraffin according to standard procedures and sectioned at $7 \mu \mathrm{m}$. Sections were dewaxed, rehydrated, and stained with Alcian blue followed by standard Hematoxylin and Eosin staining.

The QM7 cells [33] were grown in Medium 199 (Invitrogen) with Glutamax (Invitrogen) supplemented with 10\% FCS and 10\% tryptose phosphate broth. Cells were infected with concentrated viral supernatant, allowed to replicate for 3 days, and plated in equal amounts on 12-well dishes (4 wells per construct). Myogenic differentiation was induced with Medium 199 supplemented with $0.5 \%$ FCS for 1 to 7 days with daily change of medium. Muscle staining with antibody A4.1025 (Upstate) was performed using the Vector Labs ABC staining system according to the manufacturer's recommendations. For nuclear staining 4',6-Diamidine-2phenylindole (DAPI) was applied together with the secondary antibodies at 1:2,000. Quantification of staining was done by histomorphometric analysis using the AxioVision ${ }^{\circledR}$ software tool AutMess ${ }^{\mathrm{TM}}$ (Zeiss).

The BMSCs were collected from chicken fetal tibiae at day 20. The diaphysis was isolated, and bone marrow was flushed out with sterile PBS. The cell suspension was filtered through a cell strainer ( $45 \mu \mathrm{m}$, Becton Dickinson), collected by centrifugation, resuspended in minimal essential medium ( $\alpha$-MEM) (Invitrogen) supplemented with 10\% FCS, and plated out. Cells were cultivated for 7 days with daily washing to eliminate hematopoetic cells. For assays, the cells were trypsinized, and the suspension was calibrated to $5 \times 10^{4}$ cells per milliliter. About $500 \mu \mathrm{L}$ of this suspension plus $3 \mu \mathrm{L}$ of concentrated viral supernatant(s) per well were plated on 24-well plates and cultivated until reaching confluence. Osteoblast differentiation was stimulated using $\alpha$-MEM supplemented with $10 \%$ FCS, $10 \mu \mathrm{g} / \mathrm{mL}$ Ascorbic acid, and $2.5 \mathrm{mM} \beta$-Glycerophosphate. Medium was changed thrice weekly.

Alizarin red staining: cells were fixed in parafomaldehyde (PFA) for $30 \mathrm{~min}$, washed in PBS, and stained with Alizarin red $\mathrm{S}(0.5 \%$ aqueous solution) for $1 \mathrm{~h}$. Cells were washed several times and dried. Staining was histomorphometrically quantified using AxioVision software (Zeiss). Activity of alkaline phosphatase (ALP) was assessed as follows: cells were detached in ALP lysis buffer (0.1 M Glycin, pH 9.6, 1\% NP-40, $1 \mathrm{mM} \mathrm{MgCl}$, $1 \mathrm{mM} \mathrm{ZnCl} 2$ ), homogenized, and pelleted for 
$10 \mathrm{~min}$ at $4^{\circ} \mathrm{C}$ with $15,000 \mathrm{~g}$. About $100 \mu \mathrm{L}$ of supernatant per well (96-well plate) was used, $100 \mu \mathrm{L}$ of ALP substrate solution ( $2 \mathrm{mg} / \mathrm{mL}$ p-nitrophenyl phosphate in ALP reaction buffer (0.1 M Glycin, pH 9.6, $\left.1 \mathrm{mM} \mathrm{MgCl} 2,1 \mathrm{mM} \mathrm{ZnCl}_{2}\right]$ ) was added per well, and optical density was measured at $405 \mathrm{~nm}$.

\section{Quantitative PCR}

For real-time PCR, total RNA was isolated using TriFast (PEQLAB) according to manufacturer's protocol. One microgram of RNA was employed for reverse transcription via the GeneAmp ${ }^{\circledR}$ RNA PCR Kit (Applied Biosystems). Realtime PCR was done with SYBR ${ }^{\circledR}$ Green PCR Master Mix (Applied Biosystems). In 1 experiment, all reactions were performed at least as triplicates; primers for glyceraldehyde 3-phosphate dehydrogenase were used for normalization. For MM cultures, 4 different cultures were used for each of 3 experiments. For chicken limbs, 2 of each infected and control limbs were used and analyzed with at least 3 independent repetitions. Primer sequences are available on request.

\section{Results}

\section{Expression of Osr1 and Osr2 in limb CT cells}

During early embryonic development, Osr1 and Osr2 show a highly dynamic expression pattern with signals in the heart, kidney, branchial arches, and limbs in mouse and chick [13-15]. In the limb bud, Osr1 and Osr2 are expressed in the mesenchyme directly beneath the ectoderm in a pattern partially overlying the premuscle masses [15]. In the differentiated limb, Osr1 and Osr2 develop a unique expression pattern. At 9 days of chick embryo development (HH stage 35), the tissues of the limb have differentiated and are clearly demarcated both morphologically as well as by the expression of specific marker genes. Limb muscle patterning is completed, and myocytes have differentiated to multinucleated primary myotubes. Section ISH revealed that Osr1 is expressed in all ICTs, for example, between the skin and musculature or between muscles, as well as in all MCTs in hind- and forelimb (Fig. 1A). The Osr2 gene appeared to also be widely expressed in ICT cells, but showed prevalence for MCT (Fig. 1A). The Osr1 and Osr2 genes were not expressed in cells constituting dense CTs such as tendons and ligaments, as demonstrated by the nonoverlapping expression pattern with $S c x$ in those structures. Expression of Osr1 and Osr2 in MCT at HH35 was confirmed by ISH followed by Immunolabeling for the MyHC (Fig. 1B). The prevalence of Osr2 for MCT was confirmed by ISH on younger embryonic tissues. At HH32 (7.5 days), Osr1 showed a widespread expression in nonmuscle ICT, and also MCT is some but not all muscles (Fig. 1C); whereas Osr2 was expressed mostly in MCT cells. The Osr2 gene, however, was not

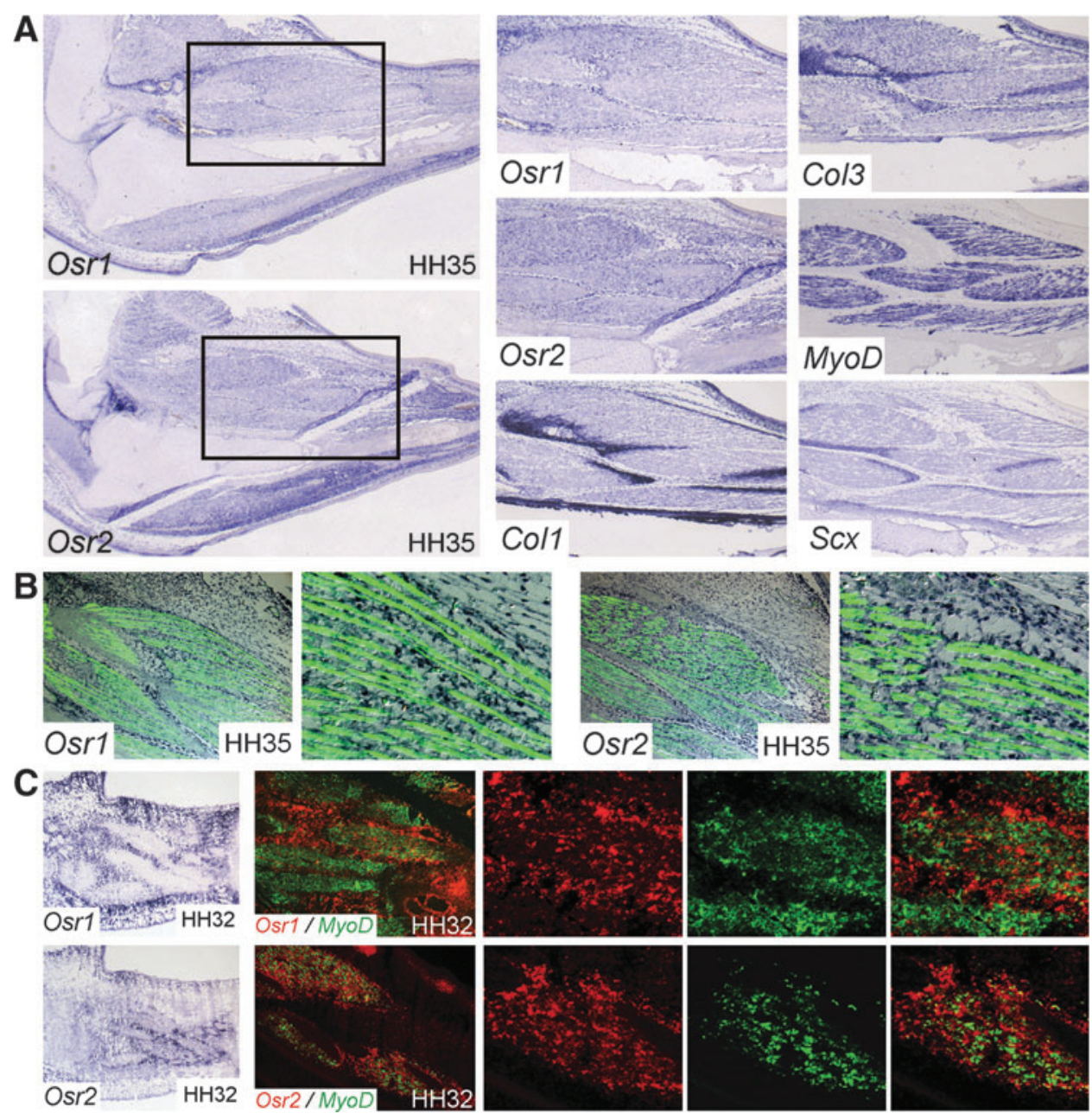

FIG. 1. Expression of Osr1 and $\mathrm{Osr} 2$ in connective tissue cells. (A) Expression patterns of chicken Osr1 and Osr2 on HH35 (8.5 days) longitudinal tibia sections demonstrate expression of Osr1 and Osr2 in irregular connective tissue. Magnifications of boxed areas shown right in comparison to Collagen 1 alpha1 (Col1), Collagen 3 alpha1 (Col3), MyoD, and Scx. (B) Longitudinal sections of a HH35 chicken forelimb stained by ISH for Osr1 or Osr2 (black color) and immunolabeled for myosin heavy chain (green fluorescence) show expression of $\mathrm{Osr}$ genes in muscle connective tissue. (C) Two-color fluorescent ISH for Osr1 or Osr2 and MyoD on HH32 (7.5 days) hindlimbs show expression of Osr1 in muscle connective tissue and other irregular connective tissues and expression of Osr2 predominantly in muscle connective tissue. 
strictly confined to MCT (Fig. 1C). Double labeling for Osr1 or Osr2 and the myogenic marker MyoD confirmed expression of Osr1 and Osr2 in MCT cells but not in myogenic precursors at HH32 in the proximal limb muscles (Fig. 1C). This also confirmed the expression of Osr2 in MCT and the more widespread expression of Osr1.

\section{Osr1 and Osr2 are expressed in unique domains in the limb but show partial overlap with progenitor pools for tendon and MCT}

We analyzed the potential of both Osr1 and Osr2 as developmental markers for ICT. We have previously shown that expression of Osr1 and Osr2 in the limb bud starts around HH22 in superficial mesenchymal cell layers and then both genes develop a distinct expression pattern [15]. We now asked the question whether Osr1 and Osr2 charac- terize separate populations of cells in early limb development. We compared the expression of Osr1 and Osr2 by 2-color fluorescent ISH at HH24 (Fig. 2A), a time point where the different tissues of the limb arise but are not yet fixed. The Osr1 gene was expressed in the proximal limb in dorsal and ventral superficial mesenchyme and also in a central domain. The Osr2 gene was expressed in a domain extending further distal and into deeper mesenchymal layers of the limb than Osr1. The only region of overlapping expression was found in superficial proximal mesenchyme. Comparison of Osr1 expression with Pax 3 demonstrated no overlap between Osr1 positive cells and migrating myogenic precursors (Fig. 2B). The expression domain of Osr1 was mostly separated from the area of the premuscle masses with the exception of the proximal ends of the Pax3 domains where Osr1 and Pax3 positive cells were found in close proximity. Conversely, Osr2 was strongly expressed in cells surrounding
FIG. 2. Expression of Osr1 and Osr2 in mesenchymal progenitor cells of the avian limb. (A-G) Two-color fluorescent ISH on chick hindlimbs at $\mathrm{HH} 24$ (4 days) with probes indicated. Boxed areas are shown as magnifications. Orientation in all pictures is proximal to the left, distal to the right, dorsal top, ventral bottom. (A) Osr1 and Osr2 show distinct expression areas with the exception of the proximal dorsal mesenchyme. (B, C) Osr1 and Osr2 are expressed exclusively with Pax3 marking myogenic precursors. $(\mathbf{D}, \mathbf{E})$ and $(\mathbf{F}, \mathbf{G})$ Osr1 and Osr2 are expressed in distinct domains but share some degree of coexpression with markers for muscle connective tissue and tendon progenitors marked by Tcf4 and Scx, respectively.

\section{A}

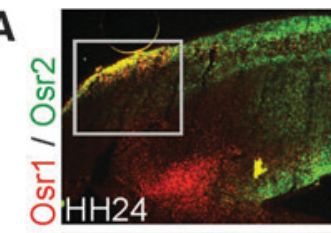

B

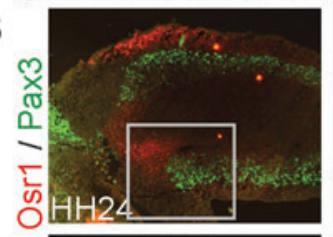

C

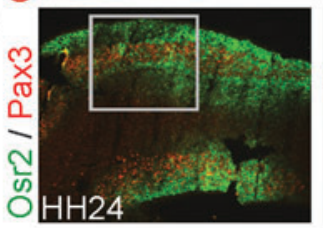

D

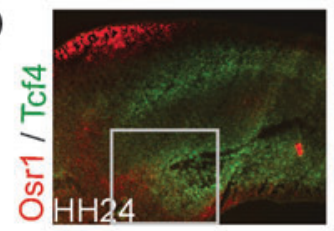

E

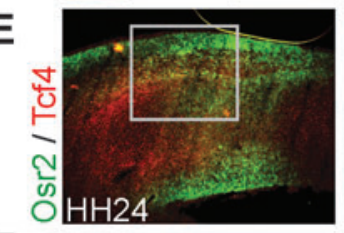

F

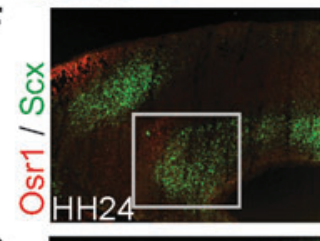

G

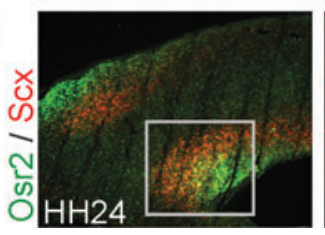

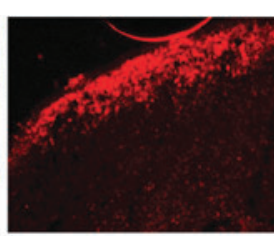
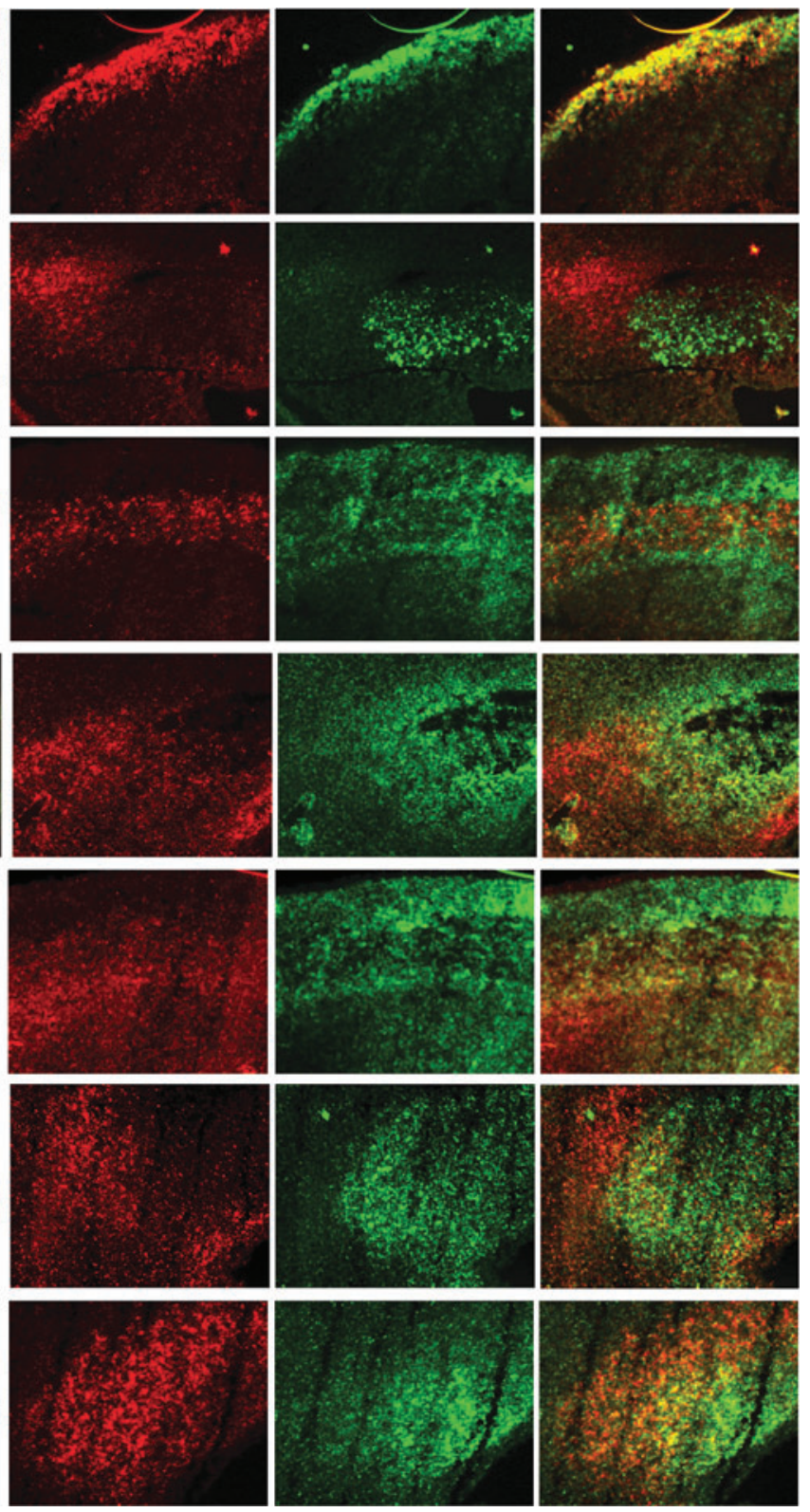
Pax3 positive myogenic precursors (Fig. 2C). Both Osr1 and Osr2 expression was not observed in Pax3 positive cells. Additionally, both Osr1 and Osr2 showed no expression in committed myoblasts expressing the myogenic differentiation factor $\mathrm{MyoD}$ at $\mathrm{HH} 24$ (not shown). Tcf4 has been described as a factor expressed in potential limb MCT progenitors [34]. At $\mathrm{HH} 24$, the domains of Osr1 and Tcf4 were distinct; however, at the proximal margin of the Tcf4 domain, they overlapped showing co-expression of both genes in a subset of cells (Fig. 2D). Interestingly, Osr2 showed a widespread overlap with Tcf4 (Fig. 2E), indicating that Osr2 also marks (albeit not exclusively) MCT precursors. Both Osr1 and Osr2 were expressed in domains largely distinct from the tendon precursor marker Scleraxis (Scx) with overlaps at the margins (Fig. 2F, G). The Osr1 and Osr2 expression was separated from Sox9positive cartilage precursors at $\mathrm{HH} 24$ (not shown); however, in the forming joints, both genes showed co-expression at the cartilage/joint interface (Supplementary Fig. S1; Supplementary Data are available online at www.liebertonline.com/scd).

\section{Osr genes influence the differentiation of mesenchymal progenitors in vitro and in vivo}

Given the expression of Osr genes in differentiated ICT and their expression pattern in the early limb largely distinct from markers for other cell populations, we hypothesized that Osr genes might play a role in ICT differentiation. To assess the role of Osr genes in mesenchymal cell differentiation, we first took advantage of the chick MM culture system. In MM cultures, undifferentiated mesenchymal cells from the chick limb bud are plated at a high density. They subsequently start to differentiate into those cell types that are present in the developing limb, that is, chondrocytes, osteoblasts, myocytes, tenocytes, and ICT cells. Chick MM cultures can be infected with a RCAS carrying a gene of interest, thus ensuring a transfection rate of almost $100 \%$ as monitored with green fluorescent protein (GFP) control infection (not shown). In MM cultures infected with either RCAS-Osr1 or RCAS-Osr2, we observed an amorphous appearance of the culture and a lower number of cartilaginous nodules. The cell mass not stained with Alcian blue had a fibroblastic appearance on sections (Fig. 3A-C). To analyze the differentiation of mesenchymal cells in culture, we performed quantitative real-time PCR (qPCR) from 5-day cultures. We used genes for ICT matrix components Collagens type IIIa1 and type VIa1 (Col3a1 and Col6a1) as markers for ICT cells. Both genes showed higher expression levels in Osr1 infected cultures than in controlGFP infected ones (Fig. 3D), indicating that the abundant fibroblastic cells we observed in Osr1-infected cultures represented ICT cells.

Additionally, endogenous Osr1 (PCR primers designed against the $3^{\prime}$ untranslated region, which is not contained in the overexpression construct) was upregulated in Osr1infected MM cultures (Fig. 3D). This was confirmed in vivo, when Osr1 was overexpressed in chicken embryonic limbs using the RCAS-system. In 3 specimens analyzed by qPCR, ICT marker genes Col3a1 and Col6a1 and endogenous Osr1 were expressed at a higher level in Osr1-infected limbs than in contralateral control limbs; however, the effect was less pronounced than in the MM cultures (Fig. 3D). To assess whether Osr genes are not only sufficient but also necessary for ICT cell differentiation, we used a RCAS-based RNAi system [28]. Three different target sequences for each, Osr1 and Osr2, were chosen and cloned into RCASARNAi. The 2 most efficient hairpin sequences for each gene were further employed in this study. Efficacy of knockdown was between $25 \%$ and $50 \%$, thus reducing expression of Osr1 or Osr2 from $50 \%$ to $75 \%$ of normal levels (Supplementary Fig. S2). The RCASARNAi carrying a sequence targeted to Gfp was used as a negative control. Knockdown of either Osr1 or Osr2 and also double knockdown of Osr1 and Osr2 resulted in a decrease in Col3a1 expression, thus indicating impaired differentiation of ICT cells (Fig. 3E). To exclude an overall influence of Osr overexpression or knockdown on cell viability and proliferation, we have used Alamar blue metabolic labeling [35] and found no differences between control cultures and cultures expressing either Osr1, Osr2, or siOsr constructs (data not shown).

Next, we analyzed whether Osr genes can interfere with the development of other mesenchymal tissues. The amount of cartilaginous condensation can be measured in MM cultures by quantification of Alcian blue incorporation into cartilaginous nodules. We found a significant antichondrogenic effect of Osr1 and Osr2 (Fig. 4A). Accordingly, overexpression of Osr1 in chicken limbs in vivo also showed thinner cartilaginous elements (Fig. 4B). Knockdown of Osr1 or Osr2 alone or in combination led to an increase in cartilage differentiation in MM cultures (Fig. 4C), indicating that endogenous Osr1 and Osr2 are necessary for the suppression of differentiation of chondrogenic cells.

In addition, overexpression of Osr1 led to a reduction of the tendon marker Scx by 2-fold in Osr1-infected MM cultures, indicative of a decrease in tendon cells (Fig. 4D). Development of tendons was also disturbed in RCAS-Osr1 infected limbs, as visualized by whole-mount ISH for $S c x$ at 9 days of development (Fig. 4E). Tendons were thinner and frayed, indicating a loss of tendon cells. In agreement with this, expression of $S c x$ was reduced in infected limbs as shown by qPCR at different time points of development (Fig. 4D). Knockdown of Osr genes in MM cultures, however, did not lead to a significant increase in Scx expression (not shown), indicating that Osr genes are not necessary for the suppression of tendon cell development.

Since Osr gene expression was never detected in myogenic cells or myotubes, we wanted to see whether misexpression of Osr genes in myogenic cells can change their commitment. We used the quail cell line QM7 [33], a committed myogenic cell line expressing the myogenic determination factor MyoD. The QM7 cells spontaneously differentiate to myotubes when switched to low-serum medium. Quail cells can be infected with the RCAS-virus, thus ensuring almost $100 \%$ transfection efficiency in the culture, as monitored by control infection with RCAS-GFP (not shown). We infected QM7 cells either with GFP-virus or with Osr1- or Osr2-virus and induced myogenic differentiation for 2 or 4 days. Myotube differentiation was assessed by staining for MyHC. In Osr1or Osr2-overexpressing cultures, there was a drastic reduction in the number of myotubes (Fig. 5A, B). The reduction in myotube number was not due to a reduction of total cell number, as demonstrated by DAPI nuclear staining (Fig. 5A). Rather, the cells between myotubes preserved a fibroblastic appearance. Thus, misexpression of Osr genes is sufficient to inhibit the default differentiation program in committed muscle progenitor cells. Similarly, in RCAS-Osr1 
FIG. 3. Osr1 and Osr2 control the differentiation of irregular connective tissue fibroblasts from mesenchymal progenitors. (A-C) Cross sections of 8 day micromass (MM) cultures infected with RCAS-Gfp (A), RCAS-Osr1 (B), or RCAS-Osr2 (C), stained with Alcian blue and hematoxylin/eosin. Note abundance of Alcian blue positive cartilage in RCAS-Gfp infected cultures versus a majority of fibroblastic cells in RCAS-

A
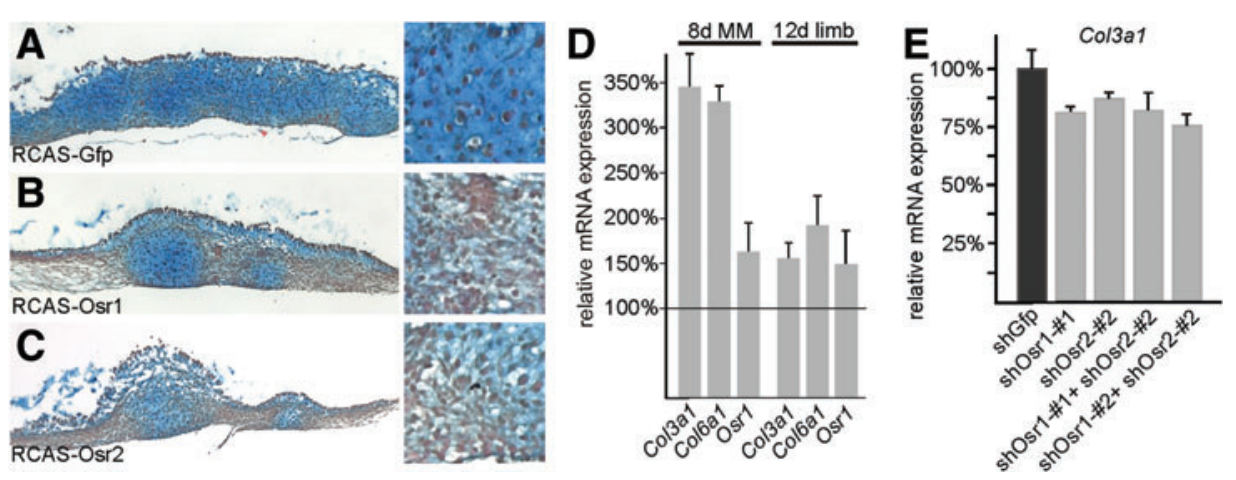

Osr1 or RCAS-Osr2 infected

cultures. (D) qPCR analysis for Col3a1, Col6a1, and endogenous Osr1 as markers for irregular connective tissue (ICT) fibroblasts on 8 day micromass cultures and 12-day-old chicken limbs infected with RCAS-Osr1. Values represent fold change of Osr1-infected sample relative to Gfp-infected control. For each marker, values combined from 3 or 2 independent experiments are shown, error bars represent standard error of the mean (SEM). (E) qPCR analysis for Col3a1 on 8 day micromass cultures treated with RCASARNAi viruses carrying indicated shRNA templates. Knockdown of Osr1 or Osr2 or combined knockdown leads to reduced expression of the ICT fibroblast marker Col3a1. Values represent fold change of shOsr1 and/or shOsr2-infected sample relative to shGfp-infected control. Mean values were determined from 3 independent experiments, error bars represent SEM. RCAS, replication competent avian retrovirus; qPCR, quantitative real-time polymerase chain reaction. Color images available online at www.liebertonline.com/scd

infected chicken wings, we observed a rarefaction of muscle fibers at day 12 of development (Fig. 5C). This coincided with an expansion of CT in concordance with the effects seen in MM cultures and the qPCR results from day 12 Osr1infected limbs (Fig. 3D). Muscle fibers generally appeared misshapen and distorted. Further, Azan blue staining for collagen fibrils demonstrated a disarray of cells and matrix in the MCT contrasting the regular array of wild type MCT cells (Fig. 5C).

\section{Osr1 and Osr2 predominantly act as transcriptional repressors in mesenchymal cell differentiation and changing their transcriptional activity can induce a reversal in mesenchymal progenitor differentiation}

Odd skipped proteins function as transcriptional repressors in Drosophila and in Xenopus [21,36]. We, thus, wanted to analyze whether a repressive or activating function can be attributed to Osr1and Osr2 in our experimental system, and
FIG. 4. Osr genes control mesenchymal cell differentiation in primary mesenchymal cell cultures and in chicken limbs. (A) Osr1 and Osr2 inhibit chondrogenesis in chicken micromass cultures. Photometric analysis of Alcian blue staining is shown for days 4, 5, and 6, representative images from day 5 cultures are shown. (B) Overexpression of Osr1 in chicken limb buds leads to a reduced size of cartilage elements. (C) Viral knockdown of Osr1 or Osr2 or in combination leads to an increase in cartilage formation in micromass cultures shown by an increase in Alcian blue incorporation (photometric measurement). (D) Real-time PCR analysis of $S c x$ as marker for tendon cells on 5 day micromass cultures and 8 and 12 days old RCAS-Osr1 infected chicken limbs. Values represent fold change of Osr1-infected sample relative to GFP-infected control. (E) Scx in situ hybridization staining on day 9 control and RCAS-Osr1 infected limb shows impaired tendon formation on Osr1 overexpression. Error bars represent standard error of the mean (SEM) from at least 3 independent experiments. Color images available online at www.liebertonline.com/scd
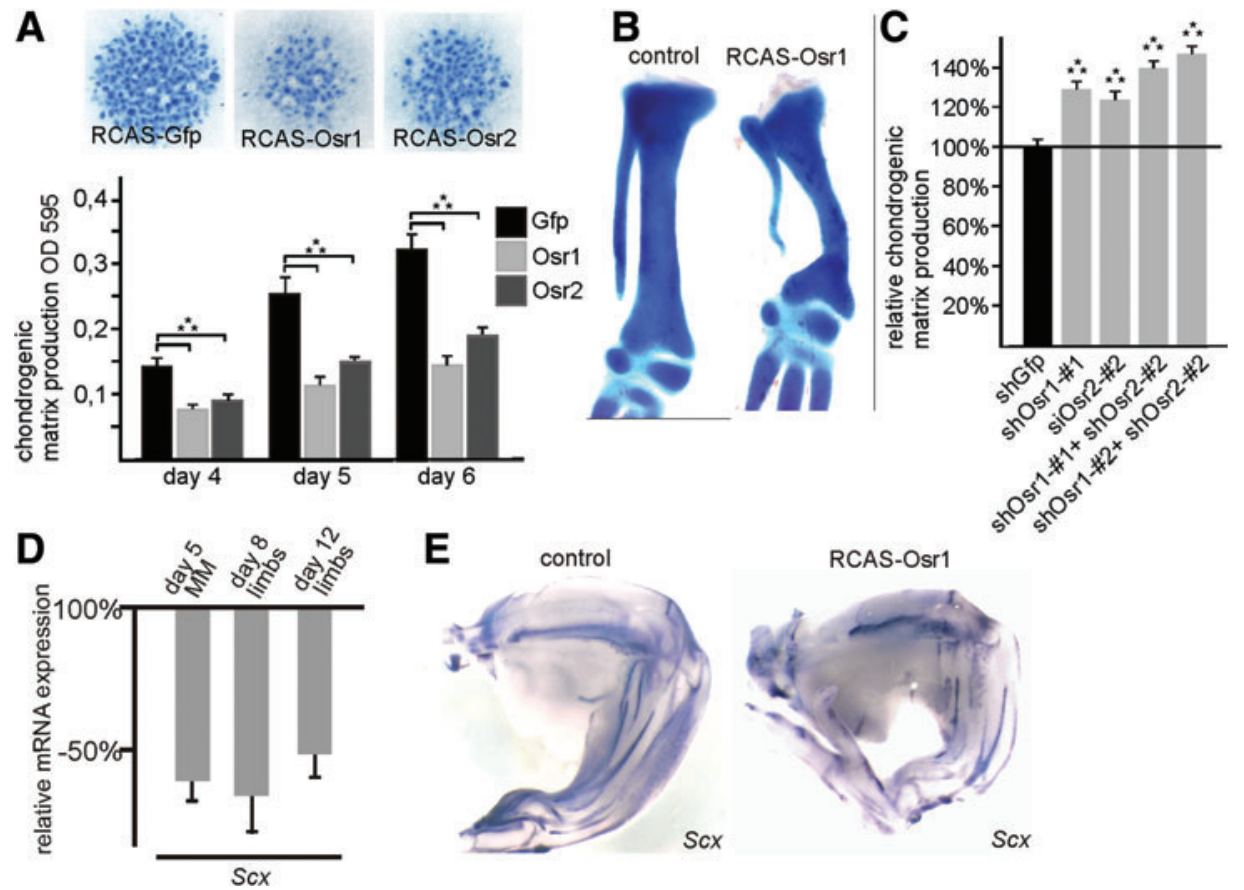

Scx 

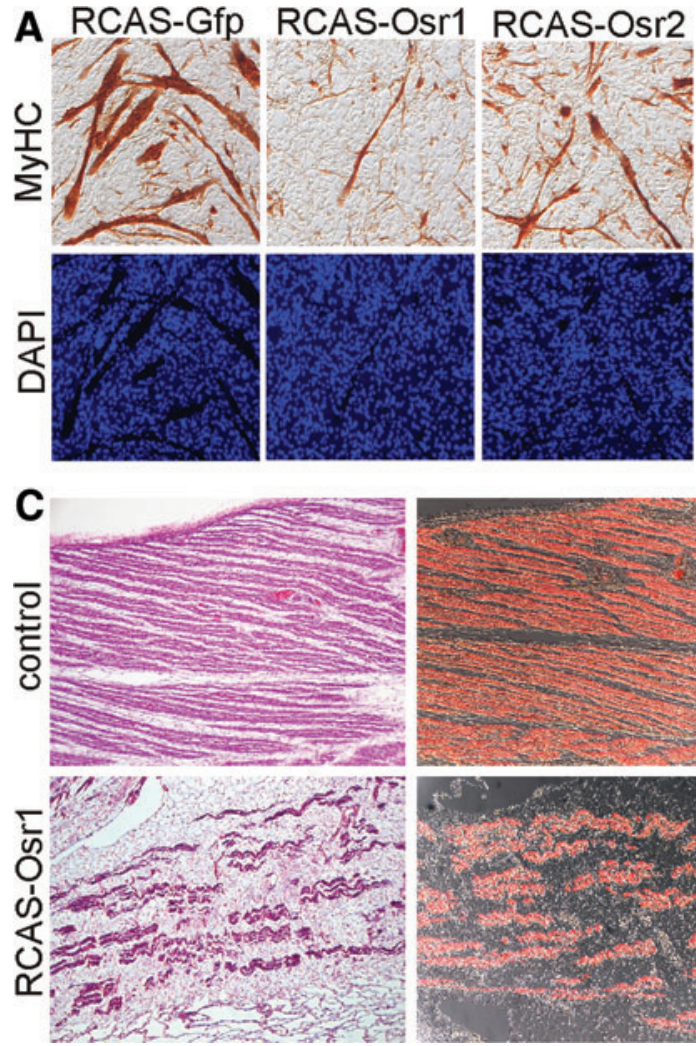
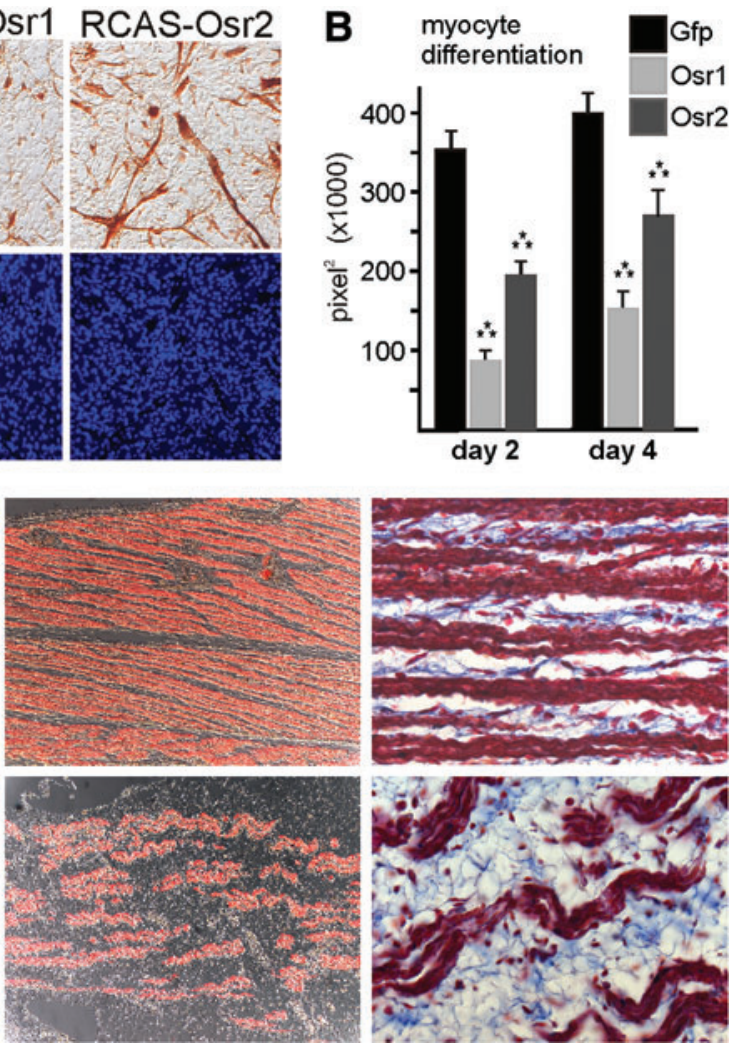

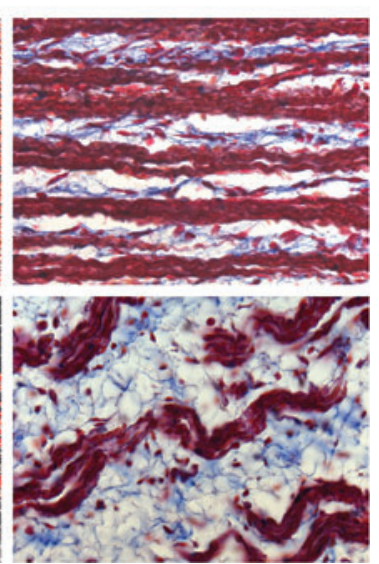

FIG. 5. Misexpression of Osr1 or Osr2 inhibits the differentiation of committed myogenic precursors. (A) QM7 myogenic cells infected with RCAS-Gfp, RCAS-Osr1, or RCASOsr2. Myogenic differentiation was induced for 2 days. Upper panel: staining for myosin heavy chain (MyHC), lower panel: 4',6-Diamidine-2-phenylindole nuclear staining. (B) Quantification of myogenic differentiation of QM7 cells by histomorphometric analysis of MyHC-stained area after 2 and 4 days of differentiation. Error bars represent standard error of the mean from at least 3 independent experiments. $t$-test: 3 asterisks: $P<0.001$. (C) Histology of RCAS-Gfp and RCAS-Osr1 infected chicken leg muscle (iliotibialis cranialis) at day 12 stained with hematoxylin and eosin (left, overview). Myosin heavy chain antibody staining combined with differential interference contrast (middle) and Azan staining for collagen fibrils (right). Note the distorted appearance and rarefaction of muscle fibers and the expansion of connective tissue. Color images available online at www.liebertonline.com/scd whether this activity is sufficient and necessary to induce the observed changes. For this purpose, we used split-function constructs harboring only the nuclear localization signal and the DNA-binding zinc finger domains of Osr1 and Osr2 fused to the engrailed-repressor (EnR) or the VP16-activator domain. These domains can, when fused to a specific DNAbinding domain, efficiently repress or activate transcription [37]. First, we analyzed MM cultures infected with RCASvirus expressing repressor or activator constructs or GFP for the expression of ICT marker genes. Expression of Col3a1 and Col6a1 was upregulated in MM cultures infected with Osr1EnR or Osr2-EnR (Fig. 6A), thus indicating an increase in ICT cells. Conversely, Osr1-VP16 or Osr2-VP16 reproducibly re- pressed the expression level of Col3a1 and Col6a1 (Fig. 6A), thus causing a decrease in ICT cells. The expression of Scx was clearly repressed by Osr1-EnR and Osr2-EnR. However, both VP16 constructs did not produce a significantly higher expression of $S c x$ in this assay in accordance with the knockdown data presented before. Using cartilage differentiation as a readout for mesenchymal cell differentiation to lineages other than ICT, we found that the repressor forms of Osr1 and Osr2 completely inhibited cartilage condensation in the MM cultures; the activator forms conversely increased the formation of cartilage (Fig. 6B).

Overall, the repressor forms of Osr1 and Osr2 phenocopied and even exacerbated the effects of the full-length proteins in
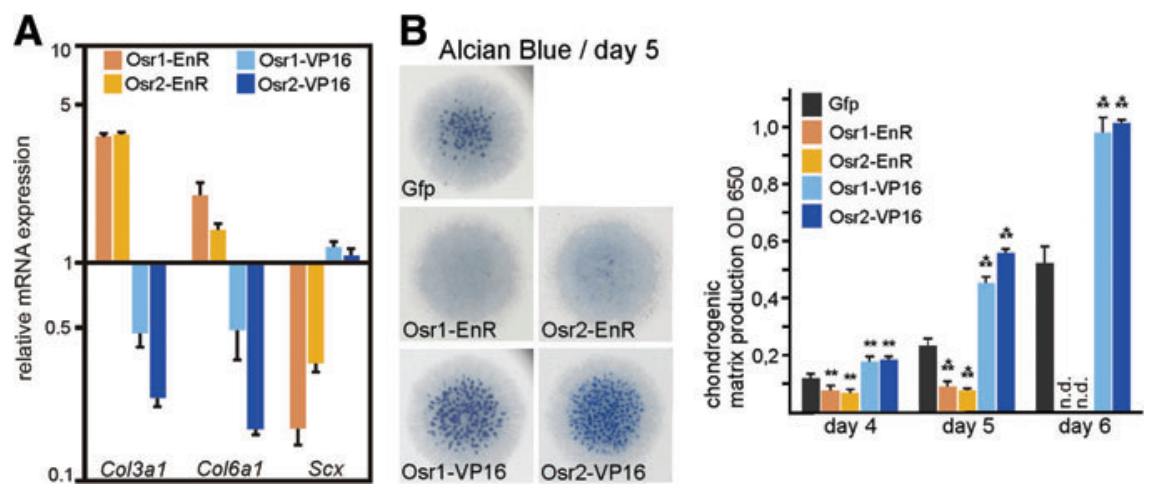
$\begin{aligned} \text { stained day } 5 \text { micromass cultures in- } & \end{aligned}$ chicken micromass cultures from days 4, 5, and 6 is shown to the right. Staining for day 6 Osr1-EnR and Osr2-EnR cultures was not determined (n.d.), as the cultures detached from the surface. Error bars represent standard error of the mean from at least 3 independent experiments. t-test: 2 asterisks: $P<0.01 ; 3$ asterisks: $P<0.001$. Color images available online at www.liebertonline.com/scd

FIG. 6. Reversal of mesenchymal cell fates by repressor and activator mutants of Osr1 and Osr2. (A) Real-time PCR analysis of 5 day micromass cultures for marker genes of connective tissue (Col3a1 and Col6a1) and tendon $(S c x)$. Cells were infected with repressor forms (Osr2-EnR, Osr2-EnR) or activator forms (Osr1-VP16, Osr2VP16). Values represent fold change of Osr1-EnR or Osr1-VP16 infected sample relative to GFP-infected control (3 independent experiments) plotted on a logarithmic scale. (B) Alcian blue 
the in vitro systems we used. On the other hand, turning Osr1 into a transcriptional activator by adding the VP16 domain resulted in a dominant-negative version of Osr1, thus leading to a reversal in mesenchymal progenitor cell differentiation when compared with wt-Osr1/2 or Osr1/2-EnR.

\section{Osr1 and Osr2 are intrinsic regulators of MSC osteogenic differentiation}

The MSCs/BMSCs are multipotent stem cells found in the adult bone marrow cavity that have the capacity to differentiate into a variety of mesenchymal tissues, for example, bone-forming osteoblasts. This capacity makes BMSCs an attractive system for regenerative medicine such as treatment of bone lesions [38,39]. Given the importance of Osr genes in embryonic mesenchymal differentiation we just described, we now aimed at analyzing the role of Osr genes in BMSC differentiation. Fourteen days after switch to osteogenic medium, Alizarin red staining demonstrated solid osteogenesis (Fig. 7A). Both Osr1 and Osr2 were capable of inhibiting osteogenesis in BMSC cultures as demonstrated by
Alizarin red staining (Fig. 7A, B) and measurement of ALP activity (Fig. 7C). Next, we used endogenous Osr1 and Osr2 and Collagen type 3a1 (Col3a1) as markers for ICT cells. qPCR showed a higher abundance of Osr1, Osr2, and Col3a1 mRNA in cultures after viral overexpression of Osr1 or Osr2 (Fig. 7D), suggesting that the BMSCs instead adopted an ICT fate. In day 10 BMSC cultures, osteogenesis visible by beginning Alizarin red staining is starting. Knockdown of either Osr1 or Osr2 alone or in combination (efficiacy see Supplementary Fig. S2) resulted in increased osteogenesis compared with control shGfp infected cultures as demonstrated by Alizarin red staining (Fig. 7E, F) and measurement of ALP activity (Fig. 7G). Thus, as in embryonic mesenchymal progenitors, Osr genes appear to enforce differentiation of BMSCs to fibroblasts while inhibiting the differentiation to other mesenchymal cell lineages such as bone-forming osteoblasts.

\section{Discussion}

ICT fibroblasts are indispensable for proper development and function of the musculoskeletal system, yet little is
FIG. 7. Osr1 and Osr2 are sufficient and necessary to control bone marrow stromal cell (BMSC) differentiation to osteoblasts. (A-D) Overexpression of Gfp (control), Osr1, or Osr2; (E-G) Viral shRNA mediated knockdown of Osr1 or $\mathrm{Osr} 2$ or both. (A) Alizarin red staining of 14 day BMSC cultures infected with the viral constructs as indicated demonstrates suppressed osteogenesis after overexpression of Osr1 or Osr2. (B) Histomorphometric quantification of Alizarin red staining from day 14 BMSC cultures. (C) Quantification of alkaline phosphatase (ALP) activity from day 14 BMSC cultures. (D) Quantitative PCR analysis of tissue marker gene expression in day 14 BMSC cultures. Osr1, Osr2: endogenous Osr genes; Col3a1: Collagen 3 type a1. (E) Alizarin red staining of day 10 BMSC cultures after knockdown of Osr1 or/ and Osr2. (F) Histomorphometric quantification of Alizarin red staining from day 10 BMSC cultures. (G) Quantification of ALP activity from day 10 BMSC cultures. Error bars represent standard error from at least 3 independent experiments. $t$-test: 3 asterisks: $P<0.001$. Color images available online at www.liebertonline.com/scd

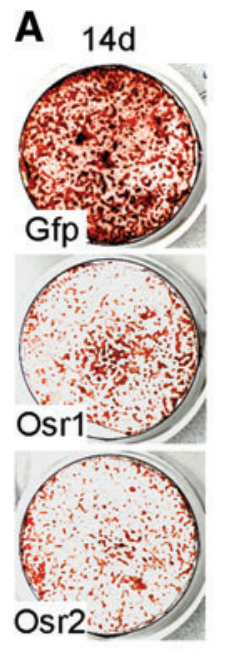

E

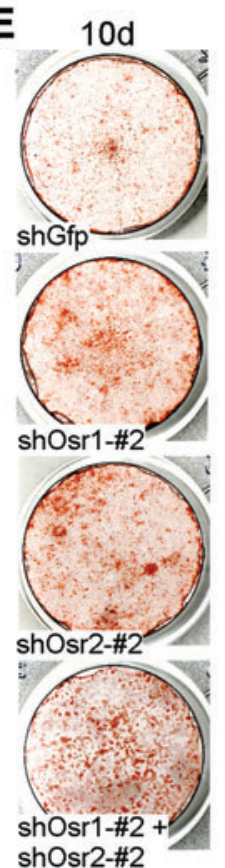

B

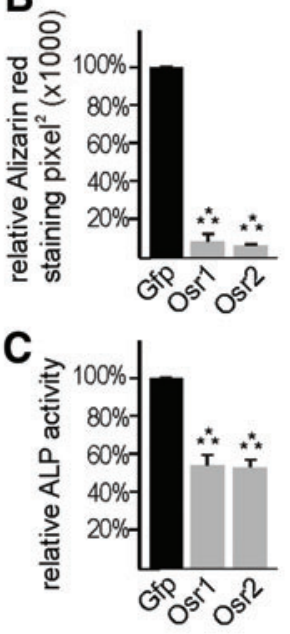

D

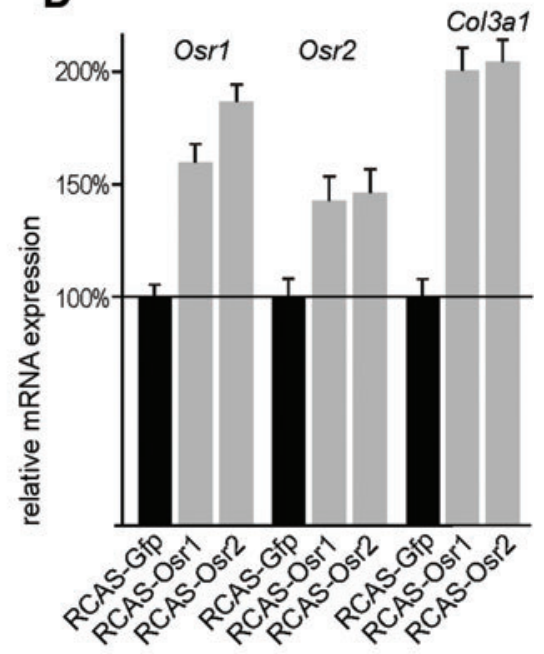

F
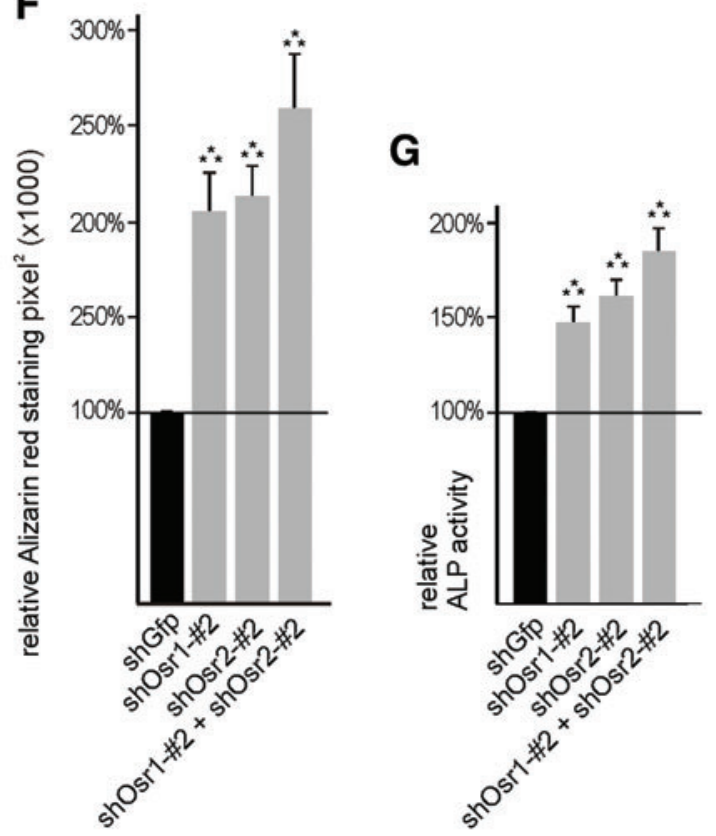
known about their developmental origin and the molecular mechanisms governing their differentiation.

The developmental analysis of ICT fibroblasts has been hampered by the lack of specific markers. We show that in the differentiated chicken limb, the expression of the zincfinger transcription factor-encoding genes $\mathrm{Osr} 1$ and $\mathrm{Osr} 2$ is highly specific for cells of ICT. However, it has to be stressed that Osr1 and Osr2 are not absolutely exclusive to ICT, as they are also expressed in other tissue types such as the synovial joints $[15,40]$ or the tendon sheaths (S. Stricker, unpublished). The initial expression of Osr1 and Osr2 can be traced down to 2 mostly separate populations of cells within the early mesenchymal limb bud. Both genes show only marginal co-expression with other mesenchymal markers, with the exception that Osr2 shows considerable coexpression with the MCT marker Tcf4 [34].

Consequently, in later development, Osr2 shows expression mostly restricted to MCT; whereas Osr1 marks all ICT cells including MCT. Remarkably, in the early limb bud putative MCT, progenitors are excluded from Osr1 expression. A possible explanation for this is that $\mathrm{CT}$ progenitors in the limb mesoderm differentiate along 2 separate lineages: an Osr1 positive population of putative nonmuscle ICT progenitors and an Osr2 and/or Tcf4 positive population of MCT progenitors. The fact that MCT at a certain time point starts to express Osr1 as well does not necessarily contradict this possibility, as the expression of Osr1 may be required at a certain step of ICT cell differentiation for specification/ maintenance of an ICT cell fate (see below). To our knowledge, Osr1 is the only available developmental marker exclusive for all ICT cells identified so far, an observation facilitating further studies needed to clarify the precise relationship of the limb CT lineage(s).

Based on the specific expression of Osr1 and Osr2 in limb ICT fibroblasts, both are likely to play a role in the specification and/or differentiation of this tissue type. We found evidence for the positive regulation of ICT cells by Osr1 and Osr2 in mesenchymal progenitor cell explant cultures or in chicken fetal limbs. Conversely, Osr1 and Osr2 were able to repress the development of other cell types such as tendon and cartilage. The latter function is in accordance with recent findings that Osr1 and Osr2 are required for the proper development of synovial joints [40]. Moreover, misexpression of Osr1 was able to inhibit the default myogenic differentiation of committed muscle progenitor cells. In comparison to the cell culture experiments, the effect of Osr1 or Osr2 on Col3 and Col6 expression was relatively weak in vivo, reflecting only a modest increase in ICT cells. Likewise, the repressive effects on tendon and cartilage were less pronounced in vivo. One possible explanation might be negative feedback mechanisms that act in vivo but are disrupted in culture systems.

The findings we report here are in analogy with functions reported for $2 \mathrm{D}$. melanogaster zinc finger transcription factors, drumstick (Drm), and Bowl, which are close relatives of Drosophila Odd skipped (Odd). In the Drosophila wing disc, Bowl controls the differentiation of the peripodial epithelium. Bowl in this context is stabilized by Drm, which is positively regulated by hedgehog signaling and negatively regulated by wingless signaling, thus integrating their reciprocal activity in peripodial versus pseudostratified columnar epithelium differentiation [41]. Interestingly, misexpression of Bowl in precursors of the pseudostratified epithelium converses these cells to peripodial epithelium, whereas inhibition of Bowl has the opposite effect [42]. In addition to that, odd-skipped family genes were also involved in regulating epithelial cellular changes during Drosophila leg segmentation, where they act downstream of the Notch signaling pathway $[43,44]$. Bowl in this context was ascribed a role in patterning specific tarsal cell fates [44]. Odd-skipped appears to be involved in analogous cell lineage decisions in vertebrates, as in Xenopus Osr1 and Osr2 are required for and can ectopically induce the differentiation of renal tissue [21]. In zebrafish, Osr1 is required for proximal pronephric nephron development, and Osr1 knockdown results in ectopic expansion of the angioblast lineage in expense of kidney precursors, whereas Osr1 overexpression resulted in the opposite cell-fate decision [45]. In mammals, Osr1 was also involved in specification of intermediate mesoderm derived lineages [22], especially renal precursors [20]. This might point to a general conserved role of oddskipped and odd-skipped-related genes in the control of tissue-type specification in different contexts.

The fact that transcriptional repressor forms of Osr1 and Osr2 showed the same effect as wild-type Osr1 or Osr2 in our assays indicates that both transcription factors act as transcriptional repressors in the context of chick ICT differentiation. In agreement with this finding, it was shown that Drosophila Odd can act as a transcriptional repressor during segmentation [36,46], and Xenopus Osr1 and Osr2 act as repressors in kidney development [21]. Moreover, a version in which the N-terminus has been replaced by a transcriptional activator domain (VP16) acts as a reverse function mutant in several test systems. It remains to be investigated how Osr1 and Osr2 accomplish this repressive effect. For Drosophila Odd, it has been shown that an eh1-like motif is required for recruiting the groucho corepressor [47]; hence, it is possible that Osr1 and Osr2 mediate their repressive activity by similar mechanisms.

Altogether, our data point to a role of Osr1 and Osr2 in the differentiation of lateral plate-derived cells of the limb. The observed changes in cell populations were observed after overexpression/misexpression or knockdown of Osr1 and/ or Osr2 demonstrated the capacity and requirement of both transcription factors to influence the fate of mesenchymal progenitor cells. However, no obvious CT defects were described in either the Osr1 or Osr2 null mutants published $[20,22,23]$; thus in the mouse, loss of either Osr1 or Osr2 appears not to be sufficient to cause changes in cellular differentiation, although subtle differences might have remained undetected. The lack of an ICT phenotype in Osr1 or Osr2 single null mutants could be due to redundancy, which is supported by genetic experiments showing that Osr1 and 2 can replace each other [18] and that only the combinatorial deletion of Osr1 and Osr2 led to joint development defects in the limb [40].

Concordant with the functions we described for Osr1 and Osr2 in mesenchymal progenitors in the embryo, we demonstrated overlapping roles for both factors in controlling the differentiation of MSCs such as BMSCs. The BMSCs have attracted immense attention as a potential therapeutic approach in a variety of degenerative conditions or injury states. Particularly in the field of bone regeneration, the application of BMSCs is an appealing option as demonstrated 
in animal models [38,39]. In this context, it is highly desirable to either predifferentiate BMSCs toward an osteoblastic commitment [48] or to be able to prevent their differentiation to other cell lineages. We show that Osr1 and Osr2 are intrinsic negative regulators of MSC differentiation to boneforming osteoblasts. It is likely that Osr factors regulate cellular differentiation by transcriptional regulation of either signaling factors or cell-cell communication molecules. Elucidating the target genes of Osr transcription factors might, thus, be a next step to improve the guided differentiation of BMSCs for regenerative therapy.

\section{Acknowledgement}

The authors thank Constance Cepko, Philippa Francis-West, Thomas Braun, and James F. Martin for probes and constructs.

\section{Author Disclosure Statement}

The authors declare that no competing financial interests exist.

\section{References}

1. Burke AC. (2007). Development and evolution of the vertebrate mesoderm. Dev Dyn 236:2369-2370.

2. Winslow BB, R Takimoto-Kimura and AC Burke. (2007). Global patterning of the vertebrate mesoderm. Dev Dyn 236:2371-2381.

3. Doljanski F. (2004). The sculpturing role of fibroblast-like cells in morphogenesis. Perspect Biol Med 47:339-356.

4. Capdevila J and JC Izpisua Belmonte. (2001). Patterning mechanisms controlling vertebrate limb development. Annu Rev Cell Dev Biol 17:87-132.

5. He L, M Papoutsi, R Huang, SI Tomarev, B Christ, H Kurz and J Wilting. (2003). Three different fates of cells migrating from somites into the limb bud. Anat Embryol 207:29-34.

6. Chevallier A, M Kieny and A Mauger. (1977). Limb-somite relationship: origin of the limb musculature. J Embryol Exp Morphol 41:245-258.

7. Christ B, HJ Jacob and M Jacob. (1977). Experimental analysis of the origin of the wing musculature in avian embryos. Anat Embryol 150:171-186.

8. Ohishi M and E Schipani. (2010). Bone marrow mesenchymal stem cells. J Cell Biochem 109:277-282.

9. Friedenstein AJ, RK Chailakhyan and UV Gerasimov. (1987). Bone marrow osteogenic stem cells: in vitro cultivation and transplantation in diffusion chambers. Cell Tissue Kinet 20:263-272.

10. Bernardo ME, F Locatelli and WE Fibbe. (2009). Mesenchymal stromal cells. Ann N Y Acad Sci 1176:101-117.

11. Bianco P, M Riminucci, S Gronthos and PG Robey. (2001). Bone marrow stromal stem cells: nature, biology, and potential applications. Stem Cells 19:180-192.

12. Kimelman N, G Pelled, Z Gazit and D Gazit. (2006). Applications of gene therapy and adult stem cells in bone bioengineering. Regen Med 1:549-561.

13. Lan Y, PD Kingsley, ES Cho and R Jiang. (2001). Osr2, a new mouse gene related to Drosophila odd-skipped, exhibits dynamic expression patterns during craniofacial, limb, and kidney development. Mech Dev 107:175-179.

14. So PL and PS Danielian. (1999). Cloning and expression analysis of a mouse gene related to Drosophila odd-skipped. Mech Dev 84:157-160.
15. Stricker S, N Brieske, J Haupt and S Mundlos. (2006). Comparative expression pattern of Odd-skipped related genes Osr1 and Osr2 in chick embryonic development. Gene Expr Patterns 6:826-834.

16. Katoh M. (2002). Molecular cloning and characterization of OSR1 on human chromosome 2p24. Int J Mol Med 10:221225.

17. Debeer P, TJ de Ravel, K Devriendt, JP Fryns, C Huysmans and WJ Van de Ven. (2002). Human homologues of Osr1 and Osr2 are not involved in a syndrome with distal limb deficiencies, oral abnormalities, and renal defects. Am J Med Genet 111:455-456.

18. Gao Y, Y Lan, CE Ovitt and R Jiang. (2009). Functional equivalence of the zinc finger transcription factors Osr1 and Osr2 in mouse development. Dev Biol 328:200-209.

19. Coulter DE and E Wieschaus. (1988). Gene activities and segmental patterning in Drosophila: analysis of odd-skipped and pair-rule double mutants. Genes Dev 2:1812-1823.

20. James RG, CN Kamei, Q Wang, R Jiang and TM Schultheiss. (2006). Odd-skipped related 1 is required for development of the metanephric kidney and regulates formation and differentiation of kidney precursor cells. Development 133:2995-3004.

21. Tena JJ, A Neto, E de la Calle-Mustienes, C Bras-Pereira, F Casares and JL Gomez-Skarmeta. (2007). Odd-skipped genes encode repressors that control kidney development. Dev Biol 301:518-531.

22. Wang Q, Y Lan, ES Cho, KM Maltby and R Jiang. (2005). Odd-skipped related 1 (Odd 1) is an essential regulator of heart and urogenital development. Dev Biol 288:582-594.

23. Lan Y, CE Ovitt, ES Cho, KM Maltby, Q Wang and R Jiang. (2004). Odd-skipped related 2 (Osr2) encodes a key intrinsic regulator of secondary palate growth and morphogenesis. Development 131:3207-3216.

24. Zhang Z, Y Lan, Y Chai and R Jiang. (2009). Antagonistic actions of Msx1 and Osr2 pattern mammalian teeth into a single row. Science 323:1232-1234.

25. Witte F, J Dokas, F Neuendorf, S Mundlos and S Stricker. (2009). Comprehensive expression analysis of all Wnt genes and their major secreted antagonists during mouse limb development and cartilage differentiation. Gene Expr Patterns 9:215-223.

26. Maggs AM, P Taylor-Harris, M Peckham and SM Hughes. (2000). Evidence for differential post-translational modifications of slow myosin heavy chain during murine skeletal muscle development. J Muscle Res Cell Motil 21:101-113.

27. Stricker S, N Verhey van Wijk, F Witte, N Brieske, K Seidel and S Mundlos. (2006). Cloning and expression pattern of chicken Ror2 and functional characterization of truncating mutations in Brachydactyly type B and Robinow syndrome. Dev Dyn 235:3456-3465.

28. Das RM, NJ Van Hateren, GR Howell, ER Farrell, FK Bangs, VC Porteous, EM Manning, MJ McGrew, K Ohyama, et al. (2006). A robust system for RNA interference in the chicken using a modified microRNA operon. Dev Biol 294:554-563.

29. Morgan BA and DM Fekete. (1996). Manipulating gene expression with replication-competent retroviruses. Methods Cell Biol 51:185-218.

30. Hamburger V and HL Hamilton. (1951). A series of normal stages in the development of the chick embryo. J Morphol 8:49-92.

31. Logan M and C Tabin. (1998). Targeted gene misexpression in chick limb buds using avian replication-competent retroviruses. Methods 14:407-420. 
32. Woods CG, S Stricker, P Seemann, R Stern, J Cox, E Sherridan, E Roberts, K Springell, S Scott, et al. (2006). Mutations in WNT7A cause a range of limb malformations, including Fuhrmann syndrome and Al-Awadi/Raas-Rothschild/ Schinzel phocomelia syndrome. Am J Hum Genet 79:402-408.

33. Antin PB and CP Ordahl. (1991). Isolation and characterization of an avian myogenic cell line. Dev Biol 143:111-121.

34. Kardon G, BD Harfe and CJ Tabin. (2003). A Tcf4-positive mesodermal population provides a prepattern for vertebrate limb muscle patterning. Dev Cell 5:937-944.

35. Rahman MS and T Tsuchiya. (2001). Enhancement of chondrogenic differentiation of human articular chondrocytes by biodegradable polymers. Tissue Eng 7:781-790.

36. Mullen JR and S DiNardo. (1995). Establishing parasegments in Drosophila embryos: roles of the odd-skipped and naked genes. Dev Biol 169:295-308.

37. Murtaugh LC, L Zeng, JH Chyung and AB Lassar. (2001). The chick transcriptional repressor Nkx3.2 acts downstream of Shh to promote BMP-dependent axial chondrogenesis. Dev Cell 1:411-422.

38. Salamon A and E Toldy. (2009). The role of adult bone marrow derived mesenchymal stem cells, growth factors and carriers in the treatment of cartilage and bone defects. J Stem Cells 4:71-80.

39. Arthur A, A Zannettino and S Gronthos. (2009). The therapeutic applications of multipotential mesenchymal/stromal stem cells in skeletal tissue repair. J Cell Physiol 218:237-245.

40. Gao Y, Y Lan, H Liu and R Jiang. (2011). The zinc finger transcription factors Osr1 and Osr2 control synovial joint formation. Dev Biol 352:83-91.

41. Hatini V, RB Green, JA Lengyel, SJ Bray and S Dinardo. (2005). The Drumstick/Lines/Bowl regulatory pathway links antagonistic Hedgehog and Wingless signaling inputs to epidermal cell differentiation. Genes Dev 19:709-718.

42. Nusinow D, L Greenberg and V Hatini. (2008). Reciprocal roles for bowl and lines in specifying the peripodial epithelium and the disc proper of the Drosophila wing primordium. Development 135:3031-3041.
43. Hao I, RB Green, O Dunaevsky, JA Lengyel and C Rauskolb. (2003). The odd-skipped family of zinc finger genes promotes Drosophila leg segmentation. Dev Biol 263:282-295.

44. de Celis Ibeas JM and SJ Bray. (2003). Bowl is required downstream of Notch for elaboration of distal limb patterning. Development 130:5943-5952.

45. Mudumana SP, D Hentschel, Y Liu, A Vasilyev and IA Drummond. (2008). Odd skipped related1 reveals a novel role for endoderm in regulating kidney versus vascular cell fate. Development 135:3355-3367.

46. Saulier-Le Drean B, A Nasiadka, J Dong and HM Krause. (1998). Dynamic changes in the functions of Odd-skipped during early Drosophila embryogenesis. Development 125:4851-4861.

47. Goldstein RE, O Cook, T Dinur, A Pisante, UC Karandikar, A Bidwai and Z Paroush. (2005). An eh1-like motif in oddskipped mediates recruitment of Groucho and repression in vivo. Mol Cell Biol 25:10711-10720.

48. Peters A, D Toben, J Lienau, H Schell, HJ Bail, G Matziolis, GN Duda and K Kaspar. (2009). Locally applied osteogenic predifferentiated progenitor cells are more effective than undifferentiated mesenchymal stem cells in the treatment of delayed bone healing. Tissue Eng Part A 15:2947-2954.

Address correspondence to: Dr. Sigmar Stricker Max Planck Institute for Molecular Genetics Ihnestrasse 73 14195 Berlin Germany

E-mail: strick_s@molgen.mpg.de

Received for publication March 30, 2011

Accepted after revision June 13, 2011

Prepublished on Liebert Instant Online June 14, 2011 